\title{
On the Final State of Black Holes
}

\section{Bo Lehnert}

Alfvén Laboratory, Royal Institute of Technology, Stockholm, Sweden

Email: bo.lehnert@ee.kth.se

How to cite this paper: Lehnert, B. (2019) On the Final State of Black Holes. Journal of Modern Physics, 10, 725-728.

https://doi.org/10.4236/jmp.2019.107052

Received: May 10, 2019

Accepted: June 17, 2019

Published: June 20, 2019

Copyright (c) 2019 by author(s) and Scientific Research Publishing Inc.

This work is licensed under the Creative Commons Attribution International License (CC BY 4.0).

http://creativecommons.org/licenses/by/4.0/

\begin{abstract}
The final state of a black hole and its force balance is considered, in the limit where all available energy is radiated away and all matter remains in the lowest form of Zero Point Energy (ZPE). In conventional theory without the concept of ZPE, the gravitational contraction would transfer the system into a mass point, being questionable from the theoretical point of view. But the inclusion of ZPE makes a force balance possible, resulting in a finite characteristic radius of a black hole mass distribution. This radius is of the same order as that of the so-called Schwarzschild radius, but is not related to the physics of the latter, which is due to particle trapping in the gravitational field.
\end{abstract}

\section{Keywords}

Black Holes, Zero Point Energy

\section{Introduction}

The black hole concept was first discovered by Schwarzschild [1] in an analysis of a simplified system having no electric charge and no angular momentum. The result was at that time merely considered as a curiosity. But a star which collapses into a black hole under the compressive action of its own gravitational field is now a subject of ever increasing interest. In its most generalized form the related physics includes both gravitational and electromagnetic fields as well as problems of General Relativity, to account for its mass, electric charge, and its intrinsic angular momentum. The associated theoretical analysis and related astronomical observations have been described in a review by Misner, Thorne and Wheeler [2]. It is thereby to be noticed that no black hole has so far been associated with a substantial electric charge.

The present investigation is limited to the Schwarzschild case of a final state of a star collapsing into a black hole. Section 2 first describes the applied physics being similar to that of the Standard Model for a vacuum state of empty space. This is followed in Section 3 by the extended analysis of a vacuum state including the Zero Point Energy 
(ZPE) discovered by Planck [3].

\section{Black Hole in a Vacuum of Empty Space}

The final state of a collapsing star and its black hole will first be considered in terms being similar to those of the Standard Model, i.e. where the vacuum is treated as an empty space where there are no sources of electric charge and current, and there is no ZPE. In this state all available energy has been radiated away. The gravitational contraction is then unbalanced, and it will continue all the way to a mathematical point. The reality of a mass point is, however, questionable from the physical point of view.

\section{Black Hole in a Vacuum Including Zero Point Energy}

When all energy of the collapsing star has been radiated away, its temperature tends to the limit $T=0$, and all matter turns into that of ZPE. The resulting mass $m$ of the black hole then corresponds to a density $\rho$ distributed inside a radius $r_{0}$ and within the volume $V=$ $\frac{4}{3} \pi r_{0}^{3}$. The average mass density is $\bar{\rho}=m / V$. We further restrict the analysis to a system without electric charge and angular momentum.

\subsection{The Inwards Pointing Gravitational Pressure}

Following Bergmann [4] a steady curl-free gravitational field strength

$$
\mathbf{g}=-\nabla \phi=\left(-\frac{d \phi}{d r}, 0,0\right) \quad\left(\mathrm{m} / \mathrm{s}^{2}\right)
$$

is considered which originates from the potential $\phi(r)$ in a spherically symmetric frame $(r, \theta, \varphi)$ where $r$ is the only independent variable. The mass density $\rho$ is the source of $\mathbf{g}$ as given by

$$
-\operatorname{div} \mathbf{g}=4 \pi G \rho=\nabla^{2} \phi=\frac{1}{r^{2}} \frac{d}{d r}\left(r^{2} \frac{d \phi}{d r}\right)
$$

where the constant of gravitation $G=6.6726 \times 10^{-11} \mathrm{~m}^{3} / \mathrm{kg} \mathrm{s}^{2}$. The associated local force density becomes

$$
\mathbf{f}=\rho \mathbf{g}
$$

The analysis is now restricted by assuming a form

$$
\phi(r)=\phi_{0}\left(r / r_{0}\right)^{\alpha}
$$

with positive values of $\alpha$. Equation (2) then yields

$$
4 \pi G \rho=\left(\phi_{0} / r_{0}^{\alpha}\right) \alpha(\alpha+1) r^{\alpha-2}>0
$$

When aiming at orders of magnitude, a further restriction to $\alpha=2$ leads to a homogeneous density $\rho=\bar{\rho}$ within the volume $0<r<r_{0}$. Then Equation (5) results in

$$
4 \pi G \rho=6 \phi_{0} / r_{0}^{2}
$$

From Equation (3)

$$
f_{r}=-2 \rho\left(\phi_{0} r_{0}^{2}\right) r=-3\left(\phi_{0} / r_{0}^{2}\right)^{2}(1 / \pi G) r \quad\left(\mathrm{~N} / \mathrm{m}^{3}\right)
$$


in combination with Equation (6). The inwards pointing gravitational pressure then becomes

$$
p_{G}=\int_{0}^{r_{0}} f_{r} d r=-\frac{3}{2}\left(\phi_{0} / r_{0}^{2}\right)^{2}(1 / \pi G) r_{0}^{2} \quad\left(\mathrm{~N} / \mathrm{m}^{2}\right)
$$

With

$$
\phi_{0} / r_{0}^{2}=\frac{2}{3} \pi G \rho=\frac{2}{3} \pi G(m / V) \quad\left(\mathrm{s}^{-2}\right)
$$

the pressure (8) takes the form

$$
p_{G}=-\frac{2}{3}(m / V)^{2} \pi G r_{0}^{2} \quad\left(\mathrm{~N} / \mathrm{m}^{2}\right)
$$

The values of the parameters $\alpha, \phi_{0}$ and $r_{0}$ represent various chosen forms for the radial distribution of mass. This freedom does not affect the order of magnitude of the results obtained here.

\subsection{The Outwards Pointing Zero Point Energy Pressure}

At the final state there is only gravitationally compressed mass $m$ at the density $\bar{\rho}$ within the region $0<r<r_{0}$ and in the form of ZPE. In the outer vacuum region $r>r_{0}$, where $r_{0}$ represents the boundary of the black hole mass, there remains a ZPE density being much lower than $\bar{\rho}$, i.e. that which would exist in the vacuum in absence of a black hole. With $\alpha=2$ and $\rho=\bar{\rho}=$ const. there is a jump in ZPE density at the boundary $r=r_{0}$. This generates an outward pointing pressure

$$
p_{Z}=m c^{2} / V \quad\left(\mathrm{~N} / \mathrm{m}^{2}\right)
$$

Even if ZPE represents the lowest energy level, its spatial gradient can thus generate a force [5].

\subsection{The Pressure Balanced Black Hole}

A pressure balance at a finite characteristic radius $r_{0}$ becomes possible through the condition $p_{G}=-p_{Z}$. Equations (10) and (11) combine to

$$
r_{0}=m G / 2 c^{2}
$$

With a solar mass of about $1.893 \times 10^{30} \mathrm{~kg}$ and a black hole of $n_{Z}$ such masses, this radius becomes

$$
r_{0}=0.7383 \times 10^{3} n_{Z}
$$

An example can be given of the black hole of the Milky Way, with a mass of about $n_{Z}=4 \times 10^{6}$. It leads to $r_{0}=3 \times 10^{9} \mathrm{~m}$, i.e. about 4 solar radii.

It should be observed that the physics of the present pressure balance differs considerably from that underlying the so called Schwarzschild radius

$$
R=2 G m / c^{2}=4 r_{0}
$$

The latter is obtained from the work which is required to "lift" a massive particle out of a black hole. The particle thereby gets trapped within the hole when being inside the radius $R$.

Equation (12) also holds approximately when not all of the gravitationally trapped matter has had time to approach zero absolute temperature $T$. 


\section{Conclusion}

Conventional physics is faced by difficulties in the treatment of black holes, in the form of an unbalanced gravitational contraction, all the way to a point mass which is questionable from the physical point of view. This can be overcome by including the effects of Zero Point Energy, the expanding force of which can outbalance that of the gravitational contraction. It results in a finite characteristic radius of the black hole configuration. This radius is of the order of the Schwarzschild radius, but not related to the physics of particle trapping in the gravitational field.

\section{Conflicts of Interest}

The author declares no conflicts of interest regarding the publication of this paper.

\section{References}

[1] Schwarzschild, K. (1916) Sitzungsber.Preuss.Akad.Wiss.Berlin (Math.Phys.), 189-196.

[2] Misner, C.W., Thorne, K.S. and Wheeler, J.A. (1973) Gravitation. Chap. 33, W.H. Freeman and Co., San Francisco and Reading.

[3] Planck, M. (1900) Verh. Dtsch Phys. Ges., 2, 237.

[4] Bergmann, P.G. (1942) Introduction to the Theory of Relativity. Chap. X, Prentice Hall, Inc., New York.

[5] Lehnert, B. (2019) Journal of Modern Physics, 10, 663-672.

https://doi.org/10.4236/jmp.2019.106047 